Relations industrielles

Industrial Relations

\title{
New Concepts in Wage Determination, édité par C.W. Taylor et F.C. Pierson, N.Y.: McGraw-Hill, 1957. 336 pp.
}

\section{Jacques St-Laurent}

Volume 13, numéro 1, janvier 1958

URI : https://id.erudit.org/iderudit/1022485ar

DOI : https://doi.org/10.7202/1022485ar

Aller au sommaire du numéro

Éditeur(s)

Département des relations industrielles de l’Université Laval

ISSN

0034-379X (imprimé)

1703-8138 (numérique)

Découvrir la revue

Citer ce compte rendu

St-Laurent, J. (1958). Compte rendu de [New Concepts in Wage Determination, édité par C.W. Taylor et F.C. Pierson, N.Y.: McGraw-Hill, 1957. 336 pp.] Relations industrielles / Industrial Relations, 13(1), 116-117.

https://doi.org/10.7202/1022485ar

Tous droits réservés (C Département des relations industrielles de l’Université Laval, 1958
Ce document est protégé par la loi sur le droit d'auteur. L'utilisation des services d'Érudit (y compris la reproduction) est assujettie à sa politique d'utilisation que vous pouvez consulter en ligne.

https://apropos.erudit.org/fr/usagers/politique-dutilisation/ 
que nuance à la conclusion que Chapin tire de ces constatations. Il souhaite très justement la mise au point d'une théorie cohérente de l'aménagement de l'espace urbain, mais il faut bien voir l'insuffisance de cette démarche du point de vue de l'application: une doctrine de l'urbanisme reste nécessaire, puisque les enseignements de la science, s'ils doivent éclairer l'urbaniste, ne peuvent lui dicter toutes ses options. Le plan d'urbanisme est en définitive un projet politique et, à ce titre, il présuppose aussi un arbitrage conscient entre les aspirations divergentes des groupes sociaux.

La seconde partie de l'ouvrage constitue un très précieux recueil des principales techniques de recherche utilisées pour établir le «dossier urbain 》 nécessaire à l'élaboration du plan d'aménagement. Chapin décrit ainsi: les techniques d'étude de l'économie de la ville, en insistant sur celles qui permettent le mieux de situer cette dernière dans son contexte régional; les méthodes de prévision de l'emploi et de la population; les instruments d'analyse de l'utilisation du sol et de la circulation.

L'auteur expose, dans une troisième partie, le processus d'élaboration du «plan d'utilisation du sol »: il nous initie par là au travail même de l'urbaniste. Disposant du «dossier urbain 》 et aussi d'un arsenal de normes techniquement fondées, celui-ci a pour mission de proposer la ligne de conduite à suivre dans la répartition sur le territoire des divers éléments de la structure urbaine (usines, édifices commerciaux, résidences, etc.).

On ne saurait recommander l'ouvrage dє. Chapin comme une oeuvre de vulgarisation, mais l'urbaniste et le chercheur appelé à collaborer avec lui y verront un excellent instrument de travail.

\section{Yves Martin}

New Concepts in Wage Determination, édité par C.W. Taylor et F.C. Pierson, N.Y.: McGraw-Hill, 1957. 336 pp.

Ce livre est le fruit des réflexions de douze économistes qui ont participé de façon active, à un moment ou l'autre de leur carrière, à l'élaboration et à la réalisation de politiques de salaire.
L'unité du volume a été assurée par une série de rencontres, s'étendant sur une période de trois ans, au cours desquelles les auteurs ont développé un cadre commun d'analyse.

On retrouve, dans sept essais originaux, une appréciation de l'état actuel de la théorie des salaires et une tentative d'approche nouvelle à la lumière des développements présents. Le livre est, de plus, l'occasion d'une réinterprétation de l'économique du travail. On y modifie les vues traditionnelles en y intégrant des inquiétudes d'ordre institutionnel. L'étude des conditions du marché - c'est la thèse des auteurs - ne suffit plus à expliquer la formation des taux de salaire: il y faut ajouter une étude du conditionnement institutionnel. Un des buts du volume est de développer ce dernier concept en précisant l'influence des coutumes sociales, des attitudes communautaires, du statut des unions et des interventions gouvernementales sur la détermination des salaires. On accorde une importance particulière au rôle social de la grande entreprise, à l'agrandissement des marchés, aux effets de l'évaluation des tâches. On reproche à la théorie traditionnelle de ne pas accorder suffisamment d'importance aux pressions sociales dans la détermination des salaires: elle ne fait que les noter au point de départ pour n'en plus tenir compte ensuite au cours de l'analyse. Mais, disent les auteurs, dans l'esprit même des entrepreneurs, le prix de la marchandise ou le taux de profit a, dans la détermination des salaires, souventes fois moins d'importance que certaines pressions sociales.

C'est dans cette optique, élaborée dans les quatre chapitres de la première partie du volume, que les auteurs abordent dans une deuxième partie l'analyse de la structure des salaires et dans une troisième partie celle du niveau général des salaires.

Dunlop suggère un aspect «interne » et 《externe » à la structure des salaires, par référence à celle qui existe à l'intérieur d'une entreprise et à celle qui existe sur le plan soit de l'industrie ou d'une économie nationale. Lester tente d'analyser les réactions de la gérance à des changements dans la structures des salajres.

Dans la dernière partie, Reynolds et Kerr s'efforcent de démêler les influen- 
ces des unions sur le niveau général des salaires.

Ce livre ne manquera pas d'intéresser un grand nombre de nos lecteurs pour de multiples raisons: les problèmes qui y sont discutés sont des problèmes contemporains: le plan des discussions est simple et logique; l'enchaînement des chapitres est aisé; l'absence d'appareils techniques d'analyse ne rebutera pas les non-initiés; la discussion, honnête et sérieuse, n'est jamais trop abstraite: des références statistiques nous raccrochent continuellement à la réalité, et les références biblographiques sont peu nombreuses et judicieusement choisies.

\section{J. St-L.}

\section{Addresses on Industrial Relations (1957} Series). Bureau of Industrial Relations, University of Michigan, Ann Arbor, Bulletin No. 25. $\$ 3.50$.

Dans cette publication sont groupées quatorze conférences données par des professeurs d'administration et des directeurs de personnel de grandes entreprises américaines. Les travaux furent présentés sous la direction du Département des Relations Industrielles de l'Université du Michigan au cours de réunions d'administrateurs dans quatre centres industriels du Michigan pendant l'année 1956-57. Le choix des sujets avait été préalablement déterminé par la décision majoritaire des participants qui délimitaient alors leurs besoins les plus urgents.

Ce groupe de chefs d'entreprise démontre un intérêt marqué pour les questions de formulation des politiques, de motivation des employés, d'amélioration des communications par la sélection du personnel de maîtrise. Cette insistance illustre la tendance actuelle qui vise à accroitre l'efficacité par une plus grande compétence. La première conférence donne le ton à la pensée commune qui anime tous les travaux. Les tendances nouvelles dans les relations humaines amènent la direction à repenser sa politique d'administration du personnel. La clé du problème, selon C.E. French, serait de développer chez l'individu un ensemble d'attitudes susceptibles de l'intégrer dans l'industrie, une volonté de produire, un sentiment d'appartenance à l'entreprise et la confiance en la direction. Pour celà, le département du personnel devra à l'avenir donner plus d'importance aux relations individuelles, effectuer plus de recherches, planifier davantage en longue période et exercer un contrôle nlus adéquat.

Les travaux sont d'inégale longueur et d'inégale valeur. Ils sont tous suivis par un résumé des discussions qui, sur le plan pratique, sont d'un réel intérêt, et parfois d'une portée appréciable. Quelques-uns s'imposent tant par leur actualité que par leur justesse: «Employee Motivation », par R.L. Kahn; "The Shorter Workweek Issue », par W. Harber et F.W. Climer; «Employee Benefits - Their Current Patterns and Trends », par J.V. Strong; «Supplemental Unemployment Benefits: Principles and Problems », par R.A. Leutheuser.

«Addresses on Industrial Relations》 est recommandé pour ceux qui sont engagés dans la direction du personnel. Ils y trouveront une source de pensée capable d'orienter leur action future.

\section{L.-M .TrEMBLAY}

